\title{
Research on Quality Inspection and Sorting On-line System for Bearing Steel Ball
}

\author{
Runxiang $\mathrm{Yu}^{1, a}$, HuiHui Sun, Jian Geng \\ ${ }^{1}$ North China Institute Of Science And Technology
}

\begin{abstract}
To detect the flaws of bearing steel ball and sort its quality on-line, a kind of online quality detecting and sorting system for bearing steel ball is developed, which based on the eddy current inspection theory. The design of scheme, circuit and mechanical structure of the system is described in detail. PSD(Phase Sensitive Detection) is applied to pick up the signal of cracks and restrain interferential signal. The flaws will be quantificational analysed based on the X-R orthogonal decomposition technology which include the size and depth information of flaws. By analysing and processing the amplitude and phase of the defect signal, the flaws can be found with high accuracy and low error-detected ratio. ARM and MCU Processor ensures the real-time ability of discriminating algorithm. The CPLD is used for generating reference signal which can be easy to get synchronous with the source signal. It has good properties of real-time to detect the quality of bearing steel ball.
\end{abstract}

Keywords.Eddy current, steel ball, phase sensitive detection, X-R orthogonal decomposition.

\section{INTRODUCTION}

Steel ball is the important part of bearing, which qualities directly influence the precision, motion performance, lifetime of ball. The defects of steel ball near the surface or near the sub-surface generally refers to the cracks, pockmark, and these defects are key factors which restrict the improving quality of steel ball. At present, since most companies use artificial detecting technique to find the defects, high false detecting rate will occur for workers' fatigue eyes.

To solve these problems, a set of quality inspection and sorting system based on eddy current, Phase Sensitive Detection is applied to pick up the signal of cracks while restrain interferential signal. ARM Processor ensures the real-time ability of discriminating algorithm. It can detect the quality of steel ball with different sizes and grades on-line and then separate them. The system meets the real-time need of determining the defects in the ball surface on-line.

\section{DETECTION PRINCPLE}

When alternating current is passed through the coil, a magnetic field is generated around the coil. When the probe is brought in close proximity to a steel ball, the eddy current is induced on the surface or sub-surface of steel ball. Then, eddy current also produce their own magnetic fields which retroact on the secondary coil and make a great impact on the magnetic fields distribution of secondary coil. The eddy current probe is a coil of wire so it contains resistance and inductive reactance when driven by alternating current. The capacitive reactance can be dropped as most eddy current probes have little capacitive reactance. Compared with the quality goods, the resistance and inductive reactance in the secondary coil will be changed in different when defective goods close to the eddy-current probe. The change of eddy current can be see form Fig.1. Through quantitative analysis of electrical impedance secondary coil, the defects will be find.

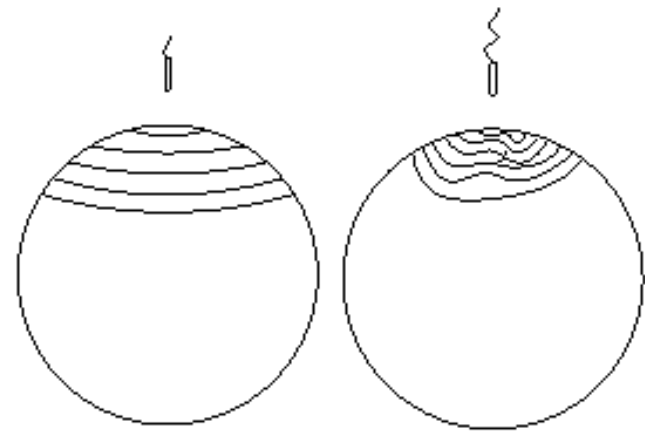

Fig.1 before and after changing when have defects

The variation of voltage in secondary coil can be described by the following equation:

$$
U=F(\mu, \sigma, x, i, n, f, r)
$$

Where:

$U$-voltage variation of coil

a yurunxiang_001@163.com 
$\mu$-magnetic permeability of measured conductor

$\sigma$-electrical conductivity of measured conductor $x$-the distance between measured conductor and detecting coil

$i$-exciting current intensity which pass though detecting coil

$n$-winding turns

$f$-Test Frequency of exciting power

$r$-shape factor of measured conductor

Once one parameter be changed while other facts fixed, the voltage of impedance signal will mapping with this parameter. Since the defects such as cracks and pocking mark have huge impact on the magnetic permeability and electrical conductivity of steel ball, the defects will be found by analysing the impedance signal which is changed by the magnetic permeability and electrical conductivity $^{[1]}$. The two key parameters of detecting coil: resistance and inductive reactance can be decomposed and the defects can be quantificational analysis based on the decomposition.

\subsection{The Decomposition Technique of Impedance Signal}

The relationship between impedance and its individual components (resistance and inductive reactance) can be represented using a vector as shown Fig. 2. The amplitude of the resistance component is shown at $\mathrm{X}$-axis and the amplitude of the inductive reactance is shown at y-axis. The amplitude of the impedance is shown by a vector that stretches from zero to a point that represents both the resistance value in the $\mathrm{x}$-direction and the inductive reactance in the $\mathrm{y}$-direction.

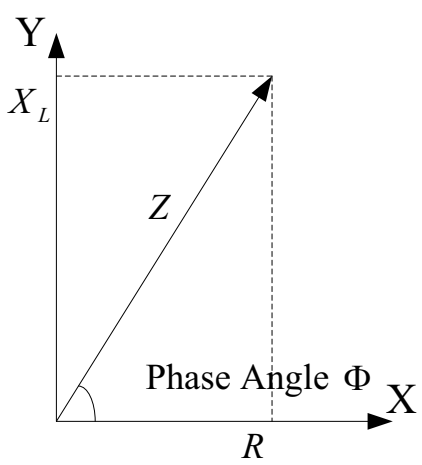

Fig.2 Impedance diagram

The impedance in a circuit with resistance and inductive reactance can be calculated using the following equation. If capacitive reactance was present in the circuit, its value would be added to the inductance term before squaring.

$$
Z=\sqrt{\left(X_{L}^{2}+R^{2}\right)}
$$

The phase angle of the circuit can be calculated using the equation below. If capacitive reactance was present in the circuit, its value would be subtracted from the inductive reactance term.

$$
\tan \Phi=X_{L} / R
$$

On propagation Process of electromagnetic wave, Phase lag is related to the time of back and forth of electromagnetic signal which enter the conductor at different depths and the back time. Phase lag is a parameter of the eddy current signal that makes it possible to obtain information about the depth of a defect within a material. The defects signal not only have amplitude, but also have phase information, so.

Phase lag change in time between the eddy current response from a disruption on the surface and a disruption at some distance below the surface. The generation of eddy currents can be thought of as a time dependent process, meaning that the eddy currents below the surface take a little longer time to form than those at the surface. Disruptions in the eddy currents away from the surface will produce more phase lag than disruptions near the surface. Phase lag is an important parameter in eddy current testing because it makes it possible to estimate the depth of a defect, and with proper reference steel ball, determining the position of a defect. The signal produced by a flaw depends on both the amplitude and phase of the eddy currents being disrupted. When decompose the coil Impedance Signal for Eddy Current Inspection of the Tube with a Crack, we can obtain the amplitude and phase of coil Impedance Signal ${ }^{[2]}$.

In this paper, Phase Sensitive Detection is applied to pick up the signal of cracks while restrain interferential signal. The two orthogonal square waves was used as the reference signal, since there is a easy way to get synchronous with the source signal from Max038. The experiments indicate that good de-noised results can be obtained and the signal-noise ratio can be improved effectively with the method. The formula is derived as follows.

The Function expression for detect signal:

$$
U_{0}=A \sin (\omega t+\Phi)
$$

The Function expression for square wave:

$$
U_{R}=\frac{4 B}{\pi}\left(\sin \omega t+\frac{1}{3} \sin 3 \omega t+\frac{1}{5} \sin 5 \omega t+\ldots\right)(5)
$$

The Function expression for square wave after the phase shift 90 degree:

$$
U_{S}=\frac{4 B}{\pi}\left(\cos \omega t-\frac{1}{3} \cos 3 \omega t+\frac{1}{5} \cos 5 \omega t-\ldots\right)
$$

After the phase sensitive detection:

$$
\begin{aligned}
& U_{X}=U_{0} \times U_{R} \\
& =\frac{2 A B}{\pi} \cos \Phi+\sum_{n=0}^{n \rightarrow \infty} \frac{-4 A B}{\pi(2 n+1)(2 n+3)} \cos [(2 n+2) \omega t+\Phi]
\end{aligned}
$$

The same:

$U_{Y}=U_{0} \times U_{S}$ 


$$
=\frac{2 A B}{\pi} \sin \Phi+\sum_{n=0}^{n \rightarrow \infty} \frac{(-1)^{n} 4 A B}{\pi(2 n+1)(2 n+3)} \cos [(2 n+2) \omega t+\Phi]
$$

After the low-pass filter, the high frequency components in the signals can be filtered, only leaving direct current components as the output:

$$
\begin{aligned}
U_{x} & =\frac{2 A B}{\pi} \cos \Phi \\
U_{y} & =\frac{2 A B}{\pi} \sin \Phi
\end{aligned}
$$

According to the theory of impedance analysis, the planar information about amplitude and phase of weak signal was achieved. It pressed noise effectively and got the two orthogonal vectors of impedance. These vectors points can be graphically displayed using the impedance plane diagram. Impedance also has an associated angle, called the phase angle of the circuit, which can be calculated by the following equation.

$$
\begin{aligned}
& \theta=\arctan \left(U_{x} / U_{y}\right) \\
& A=\sqrt{U_{x}^{2}+U_{y}^{2}}
\end{aligned}
$$

The strength of the eddy currents and the magnetic permeability of the steel ball cause the eddy current signal react on the impedance plane ${ }^{[3][4]}$, According to impedance diagram, the defects can be quantificational analysis.

\section{SYSTEM DESIGN AND EXERIMENTAL RERSULTS}

The eddy current testing system must have a signal source to generate eddy current: we use MAX038 to complete the production of excitation signals. Because of its loading capability is low, the source signal is generally amplified by the power amplifier. Since the magnetic field changes in the primary coil will also have an impact on the secondary coil. To enhance the sensitivity of detection, a voltage compensation circuit is design for removing the influence, so what the signal that the secondary coil induced is mainly come from the eddy current of the ball. The compensation circuit offset the magnetic field superimposed on secondary coil. In this condition, impedance signal of secondary coil reflects the situation of steel ball surface defect in greatest degree. The result shows that this scheme is effective in ensuring the high sensitivity. What the result with and without compensation is shown as Fig. 3.

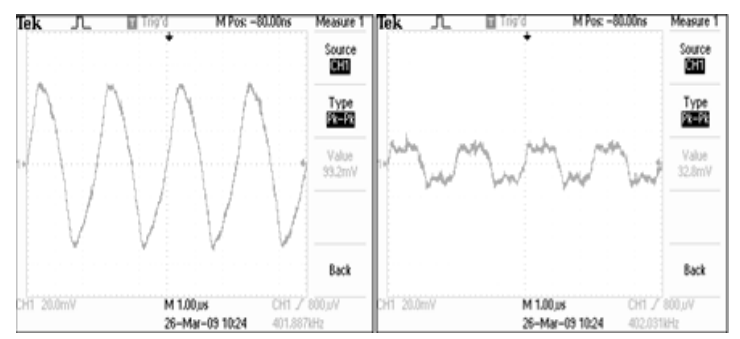

Fig.3 Secondary coil response without /with compensation
Compared the testing wave with and without compensation, the result shows that the compensative effect is good. Amplitude and phase changed obviously. Owing to weak output signals, adopts transformer coupling for the impedance matching Circuit. In mainly contains intermediate frequency transformer and preamplifying circuits. After amplification, using the phasesensitive detector orthogonal decompose for the defect signal. The CPLD takes charge of Generating two reference signal, which phase difference has 90 degree. It is easy to achieve, and facilitate synchronize with MAX038. The decomposition circuit uses two analogy multipliers to achieve phase-sensitive detector. Analogymultiplier MC1496 is the most in common use method to make two signal multiplied so that it can implement signal decomposition. It can also improve the signalnoise rate. The output of MC1496 has little frequency components, so it is easy to remove the unwanted signal through the filter circuit. The phase-sensitive detector current is design as follows Fig. 4.

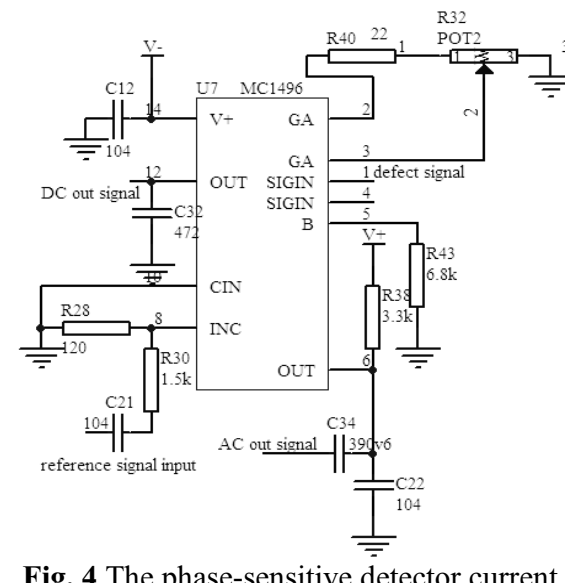

Finally we get to the X-Y signals including DC signals and AC signals. The signal is correspondence with resistance $(\mathrm{R})$, inductive reactance $(\mathrm{XL})$, The $\mathrm{DC}$ signals can assemble the impedance plane diagram ${ }^{[5]}$. If the eddy current circuit is balanced in air and then placed on a steel ball, it will lead to the DC voltage reduction. The voltage sag will change significantly when meet the defect. Most current have not collect the $\mathrm{AC}$ signal, but $\mathrm{AC}$ signal is also useful for the defect inspection. It can meet with the DC signal to enhance the responsibility of dynamic performance. The MC1496 can also make adjustment gain tuning. So it can adjust the size of the amplitude and sensitivity of DC output signal. The result of phasesensitive detector is shown on Fig. 5.

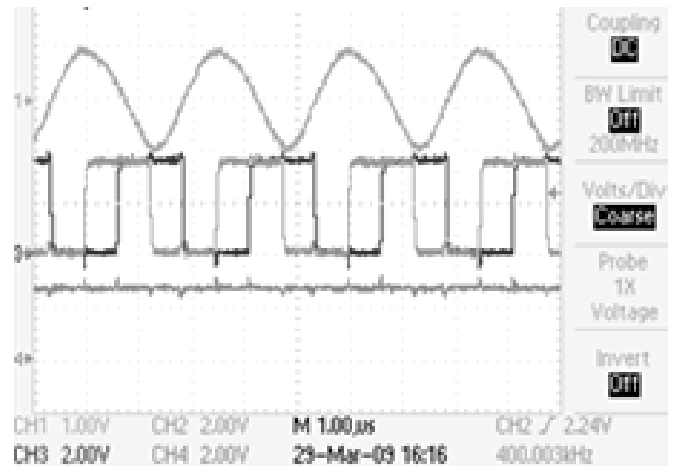


Fig. 5 Result of Phase Sensitive Detection the first wave is the weak signal, the second and third is reference signal, and the fourth is the output. The defects can be judged by the value of the DC signal or use other algorithm methods to determine the defects on the surface or near the surface of the steel ball.

The system software takes charge of man-machine interface for supervision, control and data acquisition. A software system for control based on configuring software was developed, and the process parameters such as the quantity of the good ball, bad ball, and so on. The configuration software developed by Beijing Zongheng company is easy for the ARM Processor to deal with. It possesses strong function of database, well stability for big-point number project, distributed network monitoring system. The machine interface system is shown by Fig. 6 as follows.

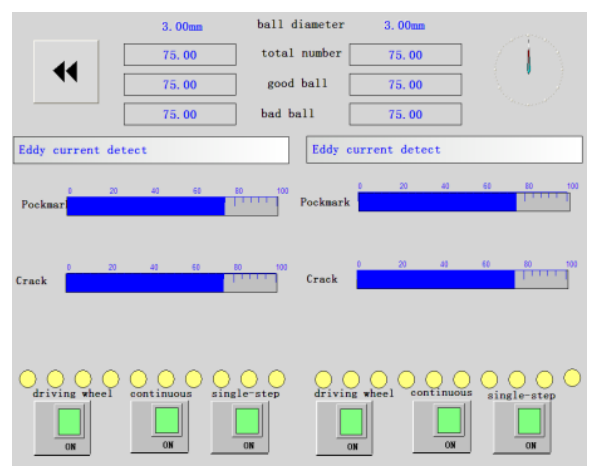

Fig. 6 Human machine interface system

\section{Conclusion}

The on-line detecting system is designed by using the principle of the eddy current. Phase Sensitive Detection is applied to pick up the signal of cracks and restrain interferential signal. ARM and MCU Processor ensures the real-time ability of discriminating algorithm. CPLD is used for generate the reference signals which have 90 degree phase difference each other, it can also improve the signal-noise rate. The upper computer software use configuration software to ensure the real-time and reliability. The results show that it can detect various kinds of typical defects such as pockmark, cracks correctly. So it has a broad prospect for development in steel ball industry.

\section{Acknowledgement}

This title is supported by the Fundamental Research

Funds for the Central Universities (3142015011).

\section{References}

1. C Belward, T Howes, and L K Forbes. "An Analytic Simplification for the Reconstruction Problem of Electrical Impedance Tomography".( 2002)

2. T. Feng, F. Lu, Y. He. Getting planar information of weak signal in dual-frequency eddy current NDT (2008)
3. P. Wu, " Phases Control in Roller Cracks Detector," Laboratory research and exploration (1999)

4. F. Yang, Z. Wu.Quantitative Indication System for Crack Depth on the Surface of Steel Material(2004)

5. M. Fan, P. Huang, Z. Zhou, " "The decomposition technique of impedance signal in eddy current testing,"(2005) 